\title{
Protein Footprinting, Conformational Dynamics and Interface-Adjacent Neutralization 'Hotspots' in the SARS-CoV-2 Spike Protein Receptor Binding Domain / Human ACE2 Interaction
}

\author{
Dominic Narang ${ }^{1}$, D. Andrew James ${ }^{2 *}$, Matthew T. Balmer ${ }^{2}$ and Derek J. Wilson ${ }^{1 *}$ \\ ${ }^{1}$ Department of Chemistry, York University, Toronto, M3J 1P3, Canada \\ ${ }^{2}$ Sanofi Pasteur Limited, 1755 Steeles Avenue West, Ontario, Toronto, Canada
}

*Corresponding authors:

Derek J. Wilson

E-mail: $\underline{\text { dkwilson@yorku.ca }}$

D. Andrew James

E-mail: Andrew.James@sanofi.com

Running Title: Footprinting the SARS-CoV-2 / ACE2 Interaction

Keywords: SARS-CoV-2, COVID-19, Spike Protein, ACE2, Hydrogen Deuterium Exchange, Footprinting, Protein Interaction 


\begin{abstract}
The novel Severe Respiratory Syndrome-like CoronaVirus (SARS-CoV-2) causes COVID-19 in humans and is responsible for one of the most destructive pandemics of the last century. At the root of SARS-CoV infection is the interaction between the viral spike protein and the human Angiotensin Converting Enzyme 2 (ACE2) protein, which allows the virus to gain entry into host cells through endocytosis. In this work, we apply Hydrogen Deuterium eXchange Mass Spectrometry (HDX-MS) to provide a detailed view of the functional footprint and conformational dynamics associated with this interaction. Our results broadly agree with the binding interface derived from high resolution X-ray crystal structure data, but also provide critical insights into shifts in structure and dynamics that accompany complexation, including some that occur immediately outside of the core binding interface. We demonstrate that these 'binding-site adjacent' dynamic shifts could represent a mechanism for the activity of a multitude of neutralizing mAbs that have been found to target these sites.
\end{abstract}




\section{INTRODUCTION}

Coronaviruses are a family of pathogens first identified by Dr. June Almeida in $1966^{1}$, characterized physically by crown-like protrusions that have the appearance of a corona. Coronaviruses are invariably highly transmissible, and most cause mild illnesses associated with the common cold. However, SARS variants, such as the SARS-CoV-1 virus that arose in 2003 can cause acute onset respiratory illness with a substantial mortality rate, especially in elderly patients. In the case of SARS-CoV-1, the overall mortality rate was $10 \%$, one of the highest ever recorded for a highly contagious respiratory virus, and upwards of $50 \%$ in patients over $60 .{ }^{2}$ Ironically, it was the high rate of mortality and morbidity that prevented SARSCoV-1 from becoming a widespread pandemic, as severe symptoms frequently developed concomitantly with transmissibility, facilitating effective isolation of transmissible individuals. However, the subsequent novel coronavirus, SARS-CoV-2, which emerged in 2019, had the necessary qualities to create a widespread pandemic, most critically a high degree of asymptomatic or weakly-symptomatic infection with significant transmissibility during the weakly-symptomatic period. ${ }^{3,4}$ Coupled to still-substantial death rates of between $0.5-1 \%$ of known infections (and again, much higher for populations over 60) and lingering effects even from mild cases, SARS-CoV-2 represents a near 'perfect-storm' pandemic virus that has wreaked havoc on social and economic networks.

At the molecular level, SARS-CoV infection is driven by a crucial interaction between the viral spike protein (homotrimers that are what give Coronavirus it's 'corona' in electron micrographs) and the human ACE2 protein, whose normal function is to catalyze the hydrolysis of the vasoconstrictor peptide angiotensin II. ${ }^{5,6}$ As determined in a $2.2 \AA$ X-ray crystal structure published in 2004 (PDB 1R42), human ACE2 is highly glycosylated, with sites at Asn-53, 90, 103, 322, 432, and 546, all likely to be high occupancy based on electron density, and has three disulfides (133-141, $344-361$ and 530 - 542). ${ }^{7}$ ACE2 is anchored to the cell membrane through a short, single-pass transmembrane domain at it's C-terminus, followed by a large extracellular sequence consisting of two domains - a collectrin-like domain and a zinc metalloproteinase domain. ${ }^{7}$ Some constructs of ACE2, including the one used in the current study, are known to dimerize in vitro, however, this does not impact the thermodynamic properties of the ACE2 / spike protein interaction. ${ }^{8}$ One of the most challenging aspects of SARS-CoV-2 infection is that ACE2 is a widely expressed protein, occurring in multiple tissues including type II alveolar cells in the lung, enterocytes in the small intestine, arterial and venous endothelial cells, arterial smooth muscle cells and cortical neurons among others. ${ }^{9}$ It is this widespread expression that is responsible for the highly varied symptoms of SARS-CoV-2 infection, including enteric illness (infection of small intestine enterocytes), increased risk of stroke (areterial/venous cell infection) and lost of taste / smell (infection of cortical neurons 
and glia). ${ }^{10}$ However, it is largely the prevalence of ACE2 in alveolar cells in the lung that drives SARSCoV mortality. ${ }^{11}$

SARS-CoV-2 spike protein is a large (1208 residue), heavily glycosylated polypeptide that forms a homotrimer in the viral capsid. Each monomer consists of two subunits (S1 and S2) with the key Receptor Binding Domain (RBD) corresponding to residues 319 - 541, which falls within the S1 subunit. ${ }^{12}$ Intact, this protein represents an insurmountable challenge to classical structural methods, however, in a demonstration of the power of the new high resolution cryo-EM technologies, the full length structure was determined to $2.8 \AA$ within months of the global onset of COVID-19 (PDB 6VXX, 6VYB). ${ }^{13}$ One of the most valuable insights to arise from these full-length structures was the occurrence of an 'open' and 'closed' configuration of the RBD relative to the rest of the protein, where only the 'open' configuration is able to efficiently bind ACE2. ${ }^{13,14}$ Important structures have also been determined by X-ray crystallography, most notably the SARS-CoV-2 spike protein RBD in complex with human ACE2 (PDB 6LZG, 6M0J). ${ }^{15}$ This wealth of structural data provide key insights into the molecular mechanisms of viral complexation and cell entry in SARS-CoV-2 infection, and can be used as a structural basis for accelerated drug and vaccine development. What they generally do not provide is a detailed picture of the shifts in conformational dynamics that also play a critical role in complexation. Adding a dynamic dimension to structural data can greatly expand the insights generated, especially in understanding allosteric effects and the subtle dynamic changes that modulate binding affinity and control specificity.

There are a limited number of analytical methods that can provide direct, structurally-resolved insights into protein conformational dynamics. The technique most closely linked to structural methods is biophysical NMR, which is uniquely powerful in the sense that it can provide direct, site specific measurements of protein dynamics. ${ }^{16}$ However, NMR suffers from a fundamental upper limit on analyte size (normally around $70 \mathrm{kDa}$, well below the size of the spike/ACE2 complex) that arises from slow rotational diffusion of large analytes. While there are some techniques that can mitigate this drawback somewhat, they are often challenging to implement. ${ }^{17}$ Hydrogen deuterium exchange mass spectrometry, in which the exchange of amide hydrogens on the protein with deuterium from solvent is measured by the increase in protein mass, is quickly becoming the most commonly used approach to measure conformational dynamics in protein interactions. ${ }^{18-22}$ The physical basis of the relationship between hydrogen deuterium exchange and protein conformation dynamics is that the observed rate of exchange depends heavily on (i) the extent to which the amide is hydrogen bonded and (ii) the degree to which the amide is accessible to solvent. ${ }^{18}$ Both of these attributes are directly impacted by conformational flexibility, linking HDX 'deuterium uptake' measurements to conformational dynamics. In the current work, we use a 'bottom up' HDX-MS workflow in which the deuterium labeling step is followed immediately by acidification (which 
quenches the exchange) and digestion of the labeled proteins by an acid protease. ${ }^{22}$ The result is a set of peptides whose deuterium uptake status reflects the extent to which their backbone amides were available for exchange in the 'native' protein, providing a degree of structural resolution to the HDX-MS experiment in segments of typically $5-10$ amino acids in length.

In this work, we examine the interaction between human ACE2 and the SARS-CoV-2 Spike RBD using HDX-MS. Our results provide a high confidence footprint for the protein/protein interface that agrees with the X-ray crystal structure and also reveals changes in conformational dynamics in functionallyrelevant regions adjacent to the binding site.

\section{METHODS}

Materials. Purified Receptor binding domain of Spike protein (SPD-C52H3) and hAce2 receptor protein (AC2-H52H8) were obtained from Acro Biosystems. Monopotassium phosphate (P0662), Dipotassium phosphate (P3786), Guanidine Hydrochloride (G7294), Tris (2-carboxyethyl)phosphine hydrochloride (TCEP, C4706), [Glub ${ }^{1}$ ]-Fibrinopeptide B human (GluFib, F3261), formic acid (33015) and Deuterium Oxide (151882) were purchased from Sigma-Aldrich. Acquity UPLC peptide CSH C18 analytical (186006934) and Vanguard Pre-column CSH C18 trap (186005303) columns were purchased from Waters. Immobilized protease XIII/pepsin (NBA2014002) column was purchased from NovaBioassays.

Hydrogen Deuterium Exchange Mass spectrometry (HDX-MS). HDX-MS experiments were performed using Waters ultra-performance liquid chromatography (nano Acquity UPLC) and Synapt G2-S mass spectrometer coupled with LEAP PAL automation technology for sample preparation as reported previously. ${ }^{23}$ The final concentration of proteins in each sample were $2.8 \mu \mathrm{M}$. For undeuterated and deuterated experiments, $7.5 \mathrm{ul}$ of protein samples were diluted in $32.5 \mathrm{ul}$ of $10 \mathrm{mM}$ potassium phosphate $150 \mathrm{mM} \mathrm{NaCl}$ buffer (Sigma-Aldrich), pH 7.5, and deuteration buffer (10 mM potassium phosphate 150 $\mathrm{mM} \mathrm{NaCl}$ (Sigma-Aldrich), pD 7.5, respectively. Five different incubation time points were used for labeling with deuterium: 0.5, 2, 1030 and 60 mins. After the dilution, the proteins samples were quenched with $40 \mathrm{ul}$ of ice-cold quench buffer (100 mM potassium phosphate, 7.5 M GdnHCl, 0.5 M TCEP (SigmaAldrich), $\mathrm{pH} 2.5,0{ }^{\circ} \mathrm{C}$ ). The quenched samples were then mixed with equal volume of $0.1 \%$ formic acid solution. One hundred microliter of the quench samples were injected into the HDX module harboring the inline pepsin protease XIII column for protein digestion. After the digestions, peptides were desalted and purified using the analytical column with a 7 min gradient of Acetonitrile with $0.1 \%$ formic acid. The purified peptides were electrosprayed into and detected by the Waters Synapt G2-Si mass spectrometer 
(MA, USA) with the mass/charge $(\mathrm{m} / \mathrm{z})$ acquisition window of 300-1700. Intermittent infusion of GluFib (785.8426 m/z, Sigma-Aldrich) was used for lock mass correction.

The peptides were identified from $\mathrm{MS}^{\mathrm{E}}$ analysis of $10 \mu \mathrm{M}$ of RBD and $5.8 \mu \mathrm{M}$ of hAce protein samples and data were analyzed using the ProteinLynx Global Server software (Waters Corp., MA, USA). The peptides identified in PLGS were then used in the DynamX 3.0 software (Waters Corp,. MA, USA). The deuterium uptake for each peptide was calculated by comparing the centroids of the mass envelopes of the deuterated samples versus the undeuterated sample. HDX data were analyzed by calculating and summing the difference in deuterium uptake for identical peptides between the two states, free and complex state of RBD and hAc2, at all the HDX time points. A back-exchange correction factor was not applied in the HDX data analysis because comparisons were made on the same peptides from both protein states. A difference of $1.5 \mathrm{Da}$ and $3 \sigma$ ( $3 \times$ standard deviation of a given peptide over the five time course) was set as the statistically significant threshold.

\section{RESULTS AND DISCUSSION}

Sequence Coverage and Differential HDX. The system explored in the current work is similar to that used in the X-ray crystal study of the SARS-CoV-2 spike protein / hACE2 complex reported recently, ${ }^{15}$ corresponding to 18 - 740 of the human ACE2 protein (Uniprot Q9BYF1-1) and 319 - 537 of the SARSCoV-2 spike protein (Uniprot QHD43416.1). A schematic depiction of the ACE2 domain structure is shown in Figure 1A with the region contained in the dashed box corresponding to the construct used. Our SARS-CoV-2 spike construct was limited specifically to the RBD (residues 319-541). Both proteins were HDX-labeled with glycosylations intact (represented by cartoon glycosyl-groups in Fig 1), with the aim of making the measurements as reflective as possible of the ACE2 / SARS-CoV-2 interaction in vivo. This approach greatly simplifies the experiment, but does come at a cost to sequence coverage. ${ }^{24}$ We were nonetheless able to achieve reasonable coverage of 77\% (ACE2) and 78.5\% (spike RBD) in differential measurements (Figure 1B, C), where peptides must be observed in both the 'free' and 'bound' datasets. Regions where coverage is conspicuously absent are, as expected, in the vicinity of known glycosylations (Figure 1B, C). Average redundancy, which reflects the extent to which overlapping peptides are detected and provides additional certainty and spatial resolution to uptake difference measurements, was relatively high at 3.78 (ACE2) and 3.98 (spike).

Importantly, peptides corresponding to all regions of the protein-protein interface as defined in the X-ray co-crystal structure were detected, including the crucial 31 - 41 and 353 - 357 regions of ACE2 and the ACE2 binding motif of spike protein (residues 437-508). All of these regions showed significantly 
reduced uptake in the bound state, with 'significance' being defined as uptake differences exceeding 1.5 $\mathrm{Da}$ and $3 \sigma$ of the sum of all timepoints, calculated with error propagation from $n=6$ technical replicates per timepoint ( $n=3$ technical replicates per state). ${ }^{25}$ Differences are heavily focused in regions within the interface, however, significant reductions in uptake are detected in 'interface-adjacent' regions. These 'adjacent' changes in conformational dynamics may once have been dismissed as functionally irrelevant consequences of the binding interaction. However there is increasing evidence that shifts in conformational dynamics occurring outside the core interface can modulate target recognition, binding specificity and even binding affinity in protein-protein interactions. ${ }^{26}$ Detecting and characterizing these shifts can therefore reveal additional sub-molecular targets for new therapeutics, indicators of potential vaccine potency or mechanistic insights for known therapeutics that bind regions outside of the interface. For instance, several neutralizing antibodies for SARS-CoV-2 spike protein isolated from COVID-19 patients (e.g., REGN10954, 10986, 1933 and 10964 among others $)^{27}$ have HDX-MS measured epitopes that include interface-adjacent regions we identify here as being impacted by ACE2 complexation.

HDX-MS footprinting of the ACE2 / SARS-CoV2-spike protein interaction. X-ray co-crystals are usually thought of as the 'gold standard' for mapping binding interfaces. ${ }^{28}$ This is because the high resolution nature of the data allows for the determination of each specific residue that is in close contact with a partner. In Figure 2A, we use the available crystal structure to map site specific contacts using a Van der Waals interaction cutoff of $4.5 \AA$ and a hydrogen bond distance cutoff maximum of $3.5 \AA$. We define the densely highlighted region along the N-terminal helix of ACE2 and the center of the spike RBD as the 'core interfacial region' (Figure 2A).

The spatial resolution of a typical bottom up HDX-MS experiment is 5 - 10 residues, depending on the size of the protein (larger proteins tend to generate longer peptides) and the extent to which the protein can be processed by the acid protease used; pepsin is relatively non-specific, but cleaves some sequences - particularly those rich in Val, Ala and Gly - substantially less efficiently. ${ }^{29}$ Spatial resolution can be improved by optimizing the digestion step (including the use of multiple acid proteases) and by using redundancy (peptide overlap) to isolate differently exchanging subsegments of each peptide. Careful optimization and use of redundancy can increase the effective resolution of bottom up HDX-MS data substantially, even to the point of allowing site-specific uptake measurements in some $\operatorname{cases}^{30}$; a feat that can also be achieved in top-down or middle-down HDX-MS. ${ }^{31}$ However, in the most straightforward implementation of the bottom up regime, like the one used here, maximal spatial resolution is traded for expediency and broad applicability, the result being the detection of binding interfaces as segment-averaged 'patches' rather than individual contacts. In the current study we detect several discontinuous regions within the ACE2 and spike sequences that exhibit a significant decrease in deuterium uptake upon 
complextion. Mapped onto the X-ray co-crystal structure (Figure 2B), these sequences correspond to a region covering the core interface, but also extending somewhat beyond it in both proteins. This raises the question of whether these 'adjacent' regions are genuinely impacted by binding, or are an artifact of the comparatively low structural resolution of HDX-MS data.

Refining the HDX-MS footprint using uptake kinetics. To get a more detailed picture of structural and dynamic changes resulting from complexation, deuterium uptake vs. labeling time profiles were plotted for all peptides (Figures S1 and S2) ${ }^{32}$, examples of which are shown in Figure 3. In general, the observed kinetic plot differences can be binned into four categories: (i) peptides that exhibit no change when in the complex, (ii) peptides that exhibit 'transient' changes in uptake when in the complex, (iii) peptides that exhibit apparently 'permanent' changes in uptake when in the complex and (iv) peptides that exhibit a mixture of type (ii) and type (iii) differences. Type (ii) differences occur when the uptake difference is exclusively the result of a decrease in the rate of deuterium uptake in the complex, with no change in the ultimate amount of uptake observed. Type (iii) differences occur when there is a decrease in the number of sites that undergo exchange in the complex (at least on the timescale of our HDX labeling step) and type (iv) differences occur when there is a change both to the rate and amount of deuterium uptake in the complex. Examples of these kinetic 'archetypes' are provided in Figure 3. Peptides exhibiting type (i) kinetic differences, which appear as purple bars in Figure 1B and C, cover by far the majority of the sequence (outside of the interfacial region) in both proteins. Peptides exhibiting type (iii) and (iv) kinetic differences are almost exclusively detected within the core of the protein-protein interface. Category (ii) kinetic differences are detected exclusively in two peptides of the spike RBD, located just outside of the core interface.

Deuterium uptake kinetics provide an additional layer of information above and beyond the uptake difference magnitudes reported in Figure 2. Specifically, close attention to uptake kinetics can yield insights into the nature of the binding interaction and/or the shifts in conformational dynamics that accompany binding. For instance, within the binding interface, relatively weak or high turnover complexation will tend to induce type (ii) kinetic differences because uptake will come to reflect the 'off' (unbound) state on the timescale of the measurement. Low turnover interactions, on the other hand will tend to cause type (iii) and/or type (iv) differences, because the 'off' state will not be significantly populated on the timescale of the measurement. In the case of the ACE2 / spike interaction, all peptides that map to the core interface exhibit type (iii) or type (iv) differences. This would imply a tight, low turnover interaction, which is consistent with the known $\mathrm{K}_{\mathrm{d}}$ of $1.2 \mathrm{nM}^{13}$

Outside of the binding interface, a type (iii) difference is indicative of a 'structural rearrangement', where complexation induces a 'permanent' reorganization of the hydrogen bond network (at least on the 
timescale of the measurement) in which one or more backbone amide hydrogens becomes entirely unavailable for exchange. We observe type (iii) differences outside the interface in a single region of ACE2, corresponding to residues $59-73$, located within an $\alpha$-helix that runs parallel and immediately adjacent to the N-terminal helix of the core interface (Figure 4A). This suggests substantial stabilization of this helix upon complexation with spike protein, or, put another way, that this helix is not exceptionally stable prior to spike binding. This helix is present, if somewhat shortened, in the ligand free crystal structure for ACE2, ${ }^{7}$ however, it is important to recognize that the crystallization process is inherently biased towards a singular, 'lowest free energy' configuration of the molecule, which often has the effect of overrepresenting what are in fact marginally stable secondary structures in solution. ${ }^{33}$

Type (ii) differences outside the binding interface are characteristic of a shift in conformational dynamics that causes one or more amide hydrogens to become less frequently available for exchange upon binding. In the current dataset, we observe such shifts in dynamics for segments $442-452$ and $471-486$, located immediately 'above' and 'below' the core interface on the spike RBD (Figure 4B). This would suggest that these 'interface adjacent' regions undergo shifts in conformational dynamics upon complexation. While it is possible that the shifts are a consequence of direct biding effects (there are a small number of distant 'direct contacts' in both regions), or are a functionally irrelevant 'side effect' of the interaction, there is a growing consensus that these shifts are often inextricably linked to the kinetic and thermodynamic properties of the interactions they accompany. If these dynamic shifts were critical, their disruption would strongly impact binding, making them potential epitopes for neutralizing antibodies in a protective immune response.

In fact, this is precisely the case: In a study in which nine neutralizing antibodies against spike protein were identified from the plasma of acute COVID-19 patients, ${ }^{27}$ virtually all of them, including the strongly neutralizing REGN10987 and REGN10933 variants, bind precisely at the 'dynamic shift' sites we identify here (Figure 4C). Given the outside position of these sites, the authors attributed neutralization to 'steric blockage' of the interface by the rest of the antibody, which is possible, but assumes a highly specific (and entirely static) orientation for the incoming antibodies. Our results suggest an alternative neutralization mechanism: That $\mathrm{mAb}$ binding in these regions prevents shifts in conformational dynamics that are an integral part of the ACE2 / spike protein interaction. Further, the consistent preference of neutralizing antibodies for these regions suggest that they provide a more effective (or perhaps more easily evolved) mechanism for neutralization in vivo than binding to the core interface itself.

\section{CONCLUSIONS}


Given the current COVID-19 crisis, there has been intensive interest in the molecular processes that drive SARS-CoV-2 infection, with antibody discovery and vaccine development occurring at an unprecedented pace. In the current study, we have examined the human ACE2 / SARS-CoV-2 spike protein interaction using hydrogen deuterium exchange mass spectrometry. As expected, this approach provided a clear footprint for the binding interface on both proteins that was entirely consistent with the cocrystal structure of the complex. Careful examination of the HDX uptake kinetics also allowed for a more detailed analysis of the binding interaction, including the significant ordering of a binding interface-adjacent helix in ACE2 and dynamic shifts adjacent to the interface on the spike protein RBD. The dynamic shifts occur in regions of the RBD that are the preferred epitopes for neutralizing antibodies targeting SARS-CoV-2 spike protein. Thus, our results support the view that, in addition to 'footprinting', HDX-MS analyses can identify 'neutralizing hotspots' outside of binding interfaces, where modulation of conformational dynamics will have a direct impact on complexation. When present, these hotspots represent additional targets for neutralizing antibodies, thereby increasing the likelyhood of a protective humoral immune response.

\section{ASSOCIATED CONTENT}

\section{Supporting Information}

The Supporting Information is available free of charge at https:// ..... Supplemental Figures 1-2 (PDF)

\section{AUTHOR INFORMATION}

\section{Corresponding Authors}

Derek J. Wilson - Department of Chemistry, York University, Toronto, ON, M3J 1P3; orcid.org/0000-0002-7012-6085; phone +1 (416) 7362100 x20786; Email: dkwilson@ yorku.ca D. Andrew James - Sanofi Pasteur Limited, 1755 Steeles Avenue West, Ontario, Toronto, Canada; https://orcid.org/0000-0002-9069-6819; phone: +1 (416) 667-2700; Email: Andrew.James@sanofi.com

\section{Authors}


Dominic Narang - Department of Chemistry, York University, Toronto, ON, M3J 1P3; https://orcid.org/0000-0003-3936-2335

Matthew T. Balmer - Sanofi Pasteur Limited, 1755 Steeles Avenue West, Ontario, Toronto, Canada

Complete contact information is available at: https:// ...

Notes

Andrew James and Matthew T. Balmer are employees of Sanofi Pasteur and may hold stock in the company.

\section{ACKNOWLEDGMENTS}

This project was supported by the Natural Sciences and Engineering Council of Canada Discovery (DG544760) and Collaborative Research and Development (CRDPJ-480432) grant programs. 


\section{REFERENCES}

(1) Almeida, J. D.; Berry, D. M.; Cunningham, C. H.; Hamre, D.; Hofstad, M. S.; Mallucci, L.; McIntosh, K.; Tyrrell, D. a. J. Coronaviruses. Nature 1968, 220 (5168), 650-+.

(2) Jiang, S.; He, Y.; Liu, S. SARS Vaccine Development. Emerg. Infect. Dis. 2005, 11 (7), 1016-1020. https://doi.org/10.3201/eid1107.050219.

(3) Petersen, E.; Koopmans, M.; Go, U.; Hamer, D. H.; Petrosillo, N.; Castelli, F.; Storgaard, M.; Al Khalili, S.; Simonsen, L. Comparing SARS-CoV-2 with SARS-CoV and Influenza Pandemics. Lancet Infect. Dis. 2020, 20 (9), e238-e244. https://doi.org/10.1016/S1473-3099(20)30484-9.

(4) Long, Q.-X.; Tang, X.-J.; Shi, Q.-L.; Li, Q.; Deng, H.-J.; Yuan, J.; Hu, J.-L.; Xu, W.; Zhang, Y.; Lv, F.-J.; Su, K.; Zhang, F.; Gong, J.; Wu, B.; Liu, X.-M.; Li, J.-J.; Qiu, J.-F.; Chen, J.; Huang, A.-L. Clinical and Immunological Assessment of Asymptomatic SARS-CoV-2 Infections. Nat. Med. 2020, 26 (8), 1200-1204. https://doi.org/10.1038/s41591-020-0965-6.

(5) Li, W.; Zhang, C.; Sui, J.; Kuhn, J. H.; Moore, M. J.; Luo, S.; Wong, S.-K.; Huang, I.-C.; Xu, K.; Vasilieva, N.; Murakami, A.; He, Y.; Marasco, W. A.; Guan, Y.; Choe, H.; Farzan, M. Receptor and Viral Determinants of SARS-Coronavirus Adaptation to Human ACE2. EMBO J. 2005, 24 (8), 16341643. https://doi.org/10.1038/sj.emboj.7600640.

(6) Li, W.; Moore, M. J.; Vasilieva, N.; Sui, J.; Wong, S. K.; Berne, M. A.; Somasundaran, M.; Sullivan, J. L.; Luzuriaga, K.; Greenough, T. C.; Choe, H.; Farzan, M. Angiotensin-Converting Enzyme 2 Is a Functional Receptor for the SARS Coronavirus. Nature 2003, 426 (6965), 450-454. https://doi.org/10.1038/nature02145.

(7) Towler, P.; Staker, B.; Prasad, S. G.; Menon, S.; Tang, J.; Parsons, T.; Ryan, D.; Fisher, M.; Williams, D.; Dales, N. A.; Patane, M. A.; Pantoliano, M. W. ACE2 X-Ray Structures Reveal a Large HingeBending Motion Important for Inhibitor Binding and Catalysis. J. Biol. Chem. 2004, 279 (17), 17996-18007. https://doi.org/10.1074/jbc.M311191200.

(8) Lui, I.; Zhou, X. X.; Lim, S. A.; Elledge, S. K.; Solomon, P.; Rettko, N. J.; Zha, B. S.; Kirkemo, L. L.; Gramespacher, J. A.; Liu, J.; Muecksch, F.; Lorenzi, J. C. C.; Schmidt, F.; Weisblum, Y.; Robbiani, D. F.; Nussenzweig, M. C.; Hatziioannou, T.; Bieniasz, P. D.; Rosenburg, O. S.; Leung, K. K.; Wells, J. A. Trimeric SARS-CoV-2 Spike Interacts with Dimeric ACE2 with Limited Intra-Spike Avidity. bioRxiv 2020, 2020.05.21.109157. https://doi.org/10.1101/2020.05.21.109157.

(9) Hamming, I.; Timens, W.; Bulthuis, M. L. C.; Lely, A. T.; Navis, G. J.; Goor, H. van. Tissue Distribution of ACE2 Protein, the Functional Receptor for SARS Coronavirus. A First Step in Understanding SARS Pathogenesis. J. Pathol. 2004, 203 (2), 631-637. https://doi.org/10.1002/path.1570.

(10) Matheson, N. J.; Lehner, P. J. How Does SARS-CoV-2 Cause COVID-19? Science 2020, 369 (6503), 510-511. https://doi.org/10.1126/science.abc6156.

(11) Wang, C.; Xie, J.; Zhao, L.; Fei, X.; Zhang, H.; Tan, Y.; Nie, X.; Zhou, L.; Liu, Z.; Ren, Y.; Yuan, L.; Zhang, Y.; Zhang, J.; Liang, L.; Chen, X.; Liu, X.; Wang, P.; Han, X.; Weng, X.; Chen, Y.; Yu, T.; Zhang, X.; Cai, J.; Chen, R.; Shi, Z.-L.; Bian, X.-W. Alveolar Macrophage Dysfunction and Cytokine Storm in the Pathogenesis of Two Severe COVID-19 Patients. EBioMedicine 2020, 57, 102833. https://doi.org/10.1016/j.ebiom.2020.102833.

(12) Ke, Z.; Oton, J.; Qu, K.; Cortese, M.; Zila, V.; McKeane, L.; Nakane, T.; Zivanov, J.; Neufeldt, C. J.; Cerikan, B.; Lu, J. M.; Peukes, J.; Xiong, X.; Kräusslich, H.-G.; Scheres, S. H. W.; Bartenschlager, R.; Briggs, J. A. G. Structures and Distributions of SARS-CoV-2 Spike Proteins on Intact Virions. Nature 2020, 1-5. https://doi.org/10.1038/s41586-020-2665-2. 
(13) Walls, A. C.; Park, Y.-J.; Tortorici, M. A.; Wall, A.; McGuire, A. T.; Veesler, D. Structure, Function, and Antigenicity of the SARS-CoV-2 Spike Glycoprotein. Cell 2020, 181 (2), 281-292.e6. https://doi.org/10.1016/j.cell.2020.02.058.

(14) Xiong, X.; Qu, K.; Ciazynska, K. A.; Hosmillo, M.; Carter, A. P.; Ebrahimi, S.; Ke, Z.; Scheres, S. H. W.; Bergamaschi, L.; Grice, G. L.; Zhang, Y.; Nathan, J. A.; Baker, S.; James, L. C.; Baxendale, H. E.; Goodfellow, I.; Doffinger, R.; Briggs, J. A. G. A Thermostable, Closed SARS-CoV-2 Spike Protein Trimer. Nat. Struct. Mol. Biol. 2020, 27 (10), 934-941. https://doi.org/10.1038/s41594-020-04785.

(15) Wang, Q.; Zhang, Y.; Wu, L.; Niu, S.; Song, C.; Zhang, Z.; Lu, G.; Qiao, C.; Hu, Y.; Yuen, K.-Y.; Wang, Q.; Zhou, H.; Yan, J.; Qi, J. Structural and Functional Basis of SARS-CoV-2 Entry by Using Human ACE2. Cell 2020, 181 (4), 894-904.e9. https://doi.org/10.1016/j.cell.2020.03.045.

(16) Kovermann, M.; Rogne, P.; Wolf-Watz, M. Protein Dynamics and Function from Solution State NMR Spectroscopy. Q. Rev. Biophys. 2016, 49, e6. https://doi.org/10.1017/S0033583516000019.

(17) Pervushin, K.; Riek, R.; Wider, G.; Wuthrich, K. Attenuated T-2 Relaxation by Mutual Cancellation of Dipole-Dipole Coupling and Chemical Shift Anisotropy Indicates an Avenue to NMR Structures of Very Large Biological Macromolecules in Solution. Proc. Natl. Acad. Sci. U. S. A. 1997, 94 (23), 12366-12371. https://doi.org/10.1073/pnas.94.23.12366.

(18) Konermann, L.; Pan, J.; Liu, Y.-H. Hydrogen Exchange Mass Spectrometry for Studying Protein Structure and Dynamics. Chem Soc Rev 2011, 40 (3), 1224-1234. https://doi.org/10.1039/COCS00113A.

(19) Oganesyan, I.; Lento, C.; Wilson, D. J. Contemporary Hydrogen Deuterium Exchange Mass Spectrometry. Methods 2018, 144, 27-42. https://doi.org/10.1016/j.ymeth.2018.04.023.

(20) Trabjerg, E.; Nazari, Z. E.; Rand, K. D. Conformational Analysis of Complex Protein States by Hydrogen/Deuterium Exchange Mass Spectrometry (HDX-MS): Challenges and Emerging Solutions. TrAC Trends Anal. Chem. 2018, 106, 125-138. https://doi.org/10.1016/j.trac.2018.06.008.

(21) Deng, B.; Lento, C.; Wilson, D. J. Hydrogen Deuterium Exchange Mass Spectrometry in Biopharmaceutical Discovery and Development - A Review. Anal. Chim. Acta 2016, 940, 8-20. https://doi.org/10.1016/j.aca.2016.08.006.

(22) Brown, K. A.; Wilson, D. J. Bottom-up Hydrogen Deuterium Exchange Mass Spectrometry: Data Analysis and Interpretation. The Analyst 2017, 142 (16), 2874-2886. https://doi.org/10.1039/C7AN00662D.

(23) Chalmers, M. J.; Busby, S. A.; Pascal, B. D.; He, Y.; Hendrickson, C. L.; Marshall, A. G.; Griffin, P. R. Probing Protein Ligand Interactions by Automated Hydrogen/Deuterium Exchange Mass Spectrometry. Anal Chem 2006, 78 (4), 1005-1014. https://doi.org/10.1021/ac051294f.

(24) Jensen, P. F.; Comamala, G.; Trelle, M. B.; Madsen, J. B.; Jorgensen, T. J. D.; Rand, K. D. Removal of N-Linked Glycosylations at Acidic PH by PNGase A Facilitates Hydrogen/Deuterium Exchange Mass Spectrometry Analysis of N-Linked Glycoproteins. Anal. Chem. 2016, 88 (24), 12479-12488. https://doi.org/10.1021/acs.analchem.6b03951.

(25) Masson, G. R.; Burke, J. E.; Ahn, N. G.; Anand, G. S.; Borchers, C.; Brier, S.; Bou-Assaf, G. M.; Engen, J. R.; Englander, S. W.; Faber, J.; Garlish, R.; Griffin, P. R.; Gross, M. L.; Guttman, M.; Hamuro, Y.; Heck, A. J. R.; Houde, D.; lacob, R. E.; Jørgensen, T. J. D.; Kaltashov, I. A.; Klinman, J. P.; Konermann, L.; Man, P.; Mayne, L.; Pascal, B. D.; Reichmann, D.; Skehel, M.; Snijder, J.; Strutzenberg, T. S.; Underbakke, E. S.; Wagner, C.; Wales, T. E.; Walters, B. T.; Weis, D. D.; Wilson, D. J.; Wintrode, P. L.; Zhang, Z.; Zheng, J.; Schriemer, D. C.; Rand, K. D. Recommendations for Performing, Interpreting and Reporting Hydrogen Deuterium Exchange Mass Spectrometry (HDX- 
MS) Experiments. Nat. Methods 2019, 16 (7), 595-602. https://doi.org/10.1038/s41592-0190459-y.

(26) Ramirez-Sarmiento, C. A.; Komives, E. A. Hydrogen-Deuterium Exchange Mass Spectrometry Reveals Folding and Allostery in Protein-Protein Interactions. Methods 2018, 144, 43-52. https://doi.org/10.1016/j.ymeth.2018.04.001.

(27) Hansen, J.; Baum, A.; Pascal, K. E.; Russo, V.; Giordano, S.; Wloga, E.; Fulton, B. O.; Yan, Y.; Koon, K.; Patel, K.; Chung, K. M.; Hermann, A.; Ullman, E.; Cruz, J.; Rafique, A.; Huang, T.; Fairhurst, J.; Libertiny, C.; Malbec, M.; Lee, W.; Welsh, R.; Farr, G.; Pennington, S.; Deshpande, D.; Cheng, J.; Watty, A.; Bouffard, P.; Babb, R.; Levenkova, N.; Chen, C.; Zhang, B.; Hernandez, A. R.; Saotome, K.; Zhou, Y.; Franklin, M.; Sivapalasingam, S.; Lye, D. C.; Weston, S.; Logue, J.; Haupt, R.; Frieman, M.; Chen, G.; Olson, W.; Murphy, A. J.; Stahl, N.; Yancopoulos, G. D.; Kyratsous, C. A. Studies in Humanized Mice and Convalescent Humans Yield a SARS-CoV-2 Antibody Cocktail. Science 2020, 369 (6506), 1010-1014. https://doi.org/10.1126/science.abd0827.

(28) Krissinel, E.; Henrick, K. Inference of Macromolecular Assemblies from Crystalline State. J. Mol. Biol. 2007, 372 (3), 774-797. https://doi.org/10.1016/j.jmb.2007.05.022.

(29) Fruton, J. S. A History of Pepsin and Related Enzymes. Q. Rev. Biol. 2002, 77 (2), 127-147. https://doi.org/10.1086/340729.

(30) Keppel, T. R.; Weis, D. D. Mapping Residual Structure in Intrinsically Disordered Proteins at Residue Resolution Using Millisecond Hydrogen/Deuterium Exchange and Residue Averaging. J. Am. Soc. Mass Spectrom. 2015, 26 (4), 547-554. https://doi.org/10.1007/s13361-014-1033-6.

(31) Landgraf, R. R.; Chalmers, M. J.; Griffin, P. R. Automated Hydrogen/Deuterium Exchange Electron Transfer Dissociation High Resolution Mass Spectrometry Measured at Single-Amide Resolution. J. Am. Soc. Mass Spectrom. 2012, 23 (2), 301-309. https://doi.org/10.1007/s13361-011-0298-2.

(32) Lumpkin, R.; Komives, E. A. DECA, a Comprehensive, Automatic Post-Processing Program for HDXMS Data. Mol. Cell. Proteomics 2019. https://doi.org/10.1074/mcp.TIR119.001731.

(33) Nielsen, J. T.; Mulder, F. A. A. Quality and Bias of Protein Disorder Predictors. Sci. Rep. 2019, 9 (1), 5137. https://doi.org/10.1038/s41598-019-41644-w. 


\section{Figure 1}

\section{(a)}

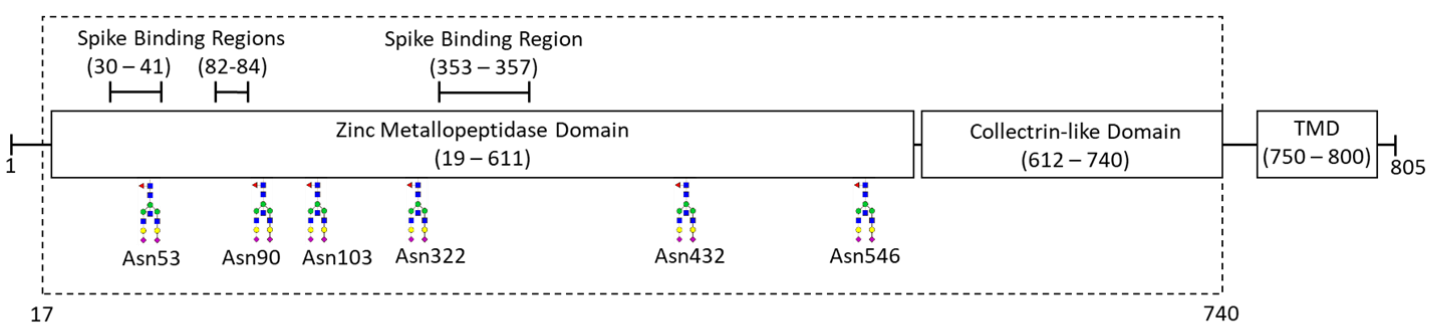

(b)

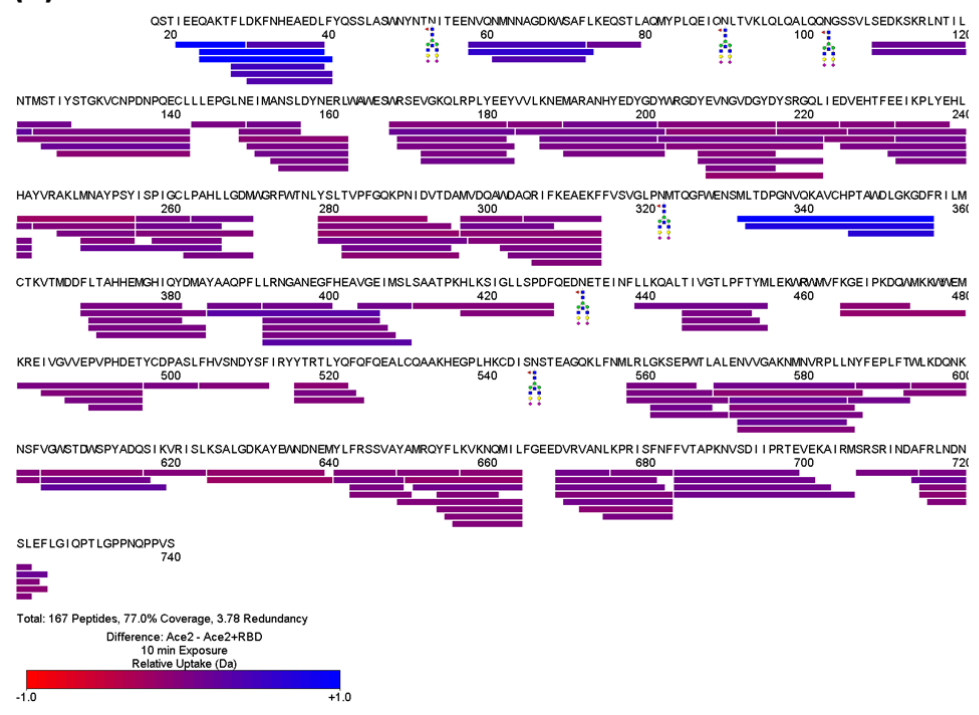

(c)

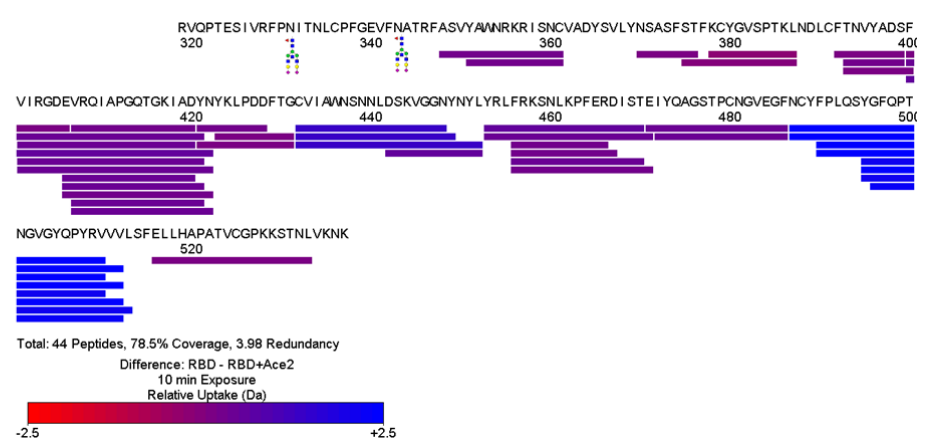

Figure 1. (a) The domain structure of human ACE2 with glycosylations and spike binding regions noted. The dotted box indicates the construct used in this study. (b) Sequence coverage map of human ACE2 with glycosylation sites and uptake differences between the free and RBD-bound state of 10 min time point noted. (c) Sequence coverage of RBD of the SARS-CoV-2 spike protein with glysocylation sites and uptake differences between the free and ACE2-bound protein for 10 min time point noted. 
Figure 2

(a)

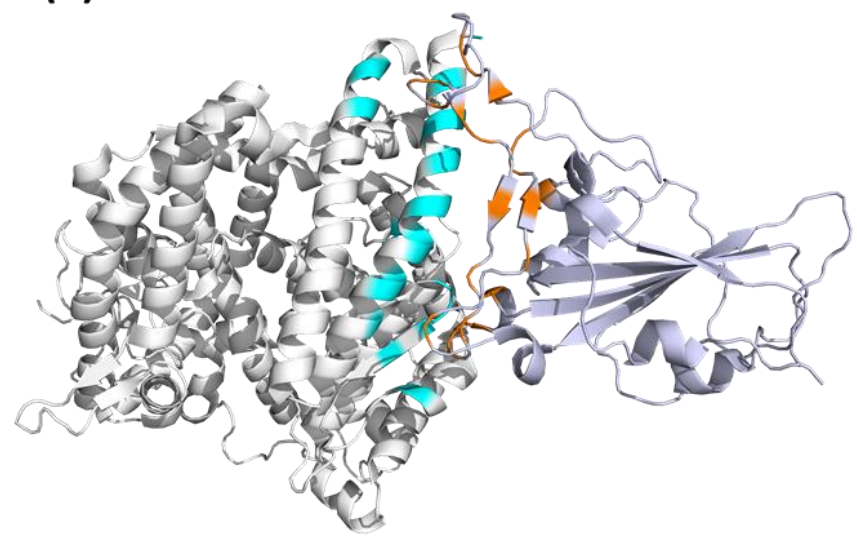

(b)

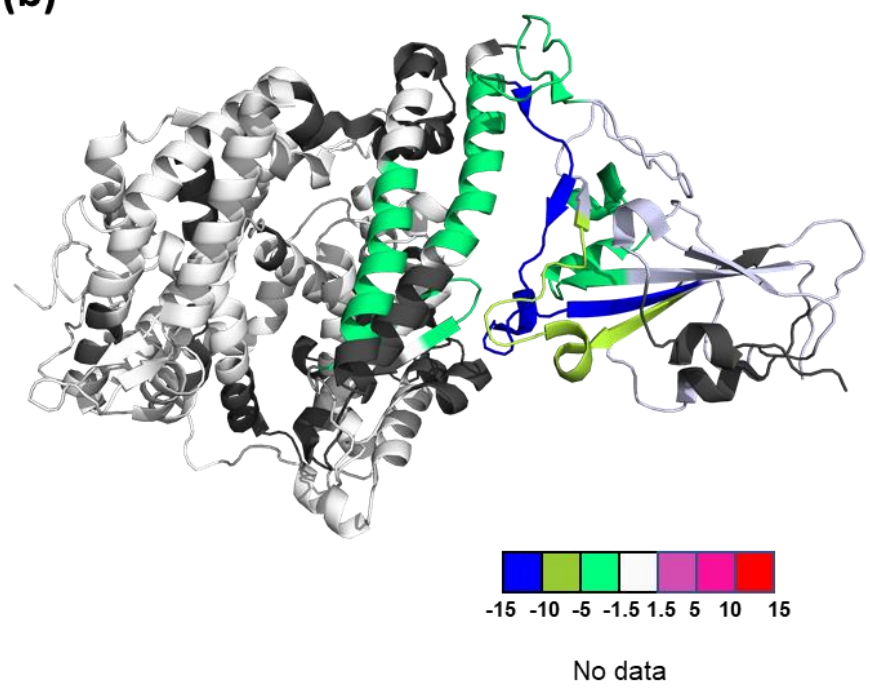

Figure 2. (a) The 'core interfacial region' of the human ACE2 / SARS-CoV-2 spike RBD interaction as determined from PDB $6 \mathrm{LZG}^{15}$ using Van der Waals interaction cutoff of $4.5 \AA$ and a hydrogen bond distance cutoff maximum of $3.5 \AA$. Contact sites are denoted in cyan and orange on ACE2 and spike RBD, respectively. (b) HDX-MS footprint of the human ACE2/SARS-CoV-2 spike interaction based on 'sum of all HDX timepoint differences' in uptake. Regions with significant uptake differences are differentiated by uptake difference magnitude (Da) using the colour scheme given in the legend. 
Figure 3

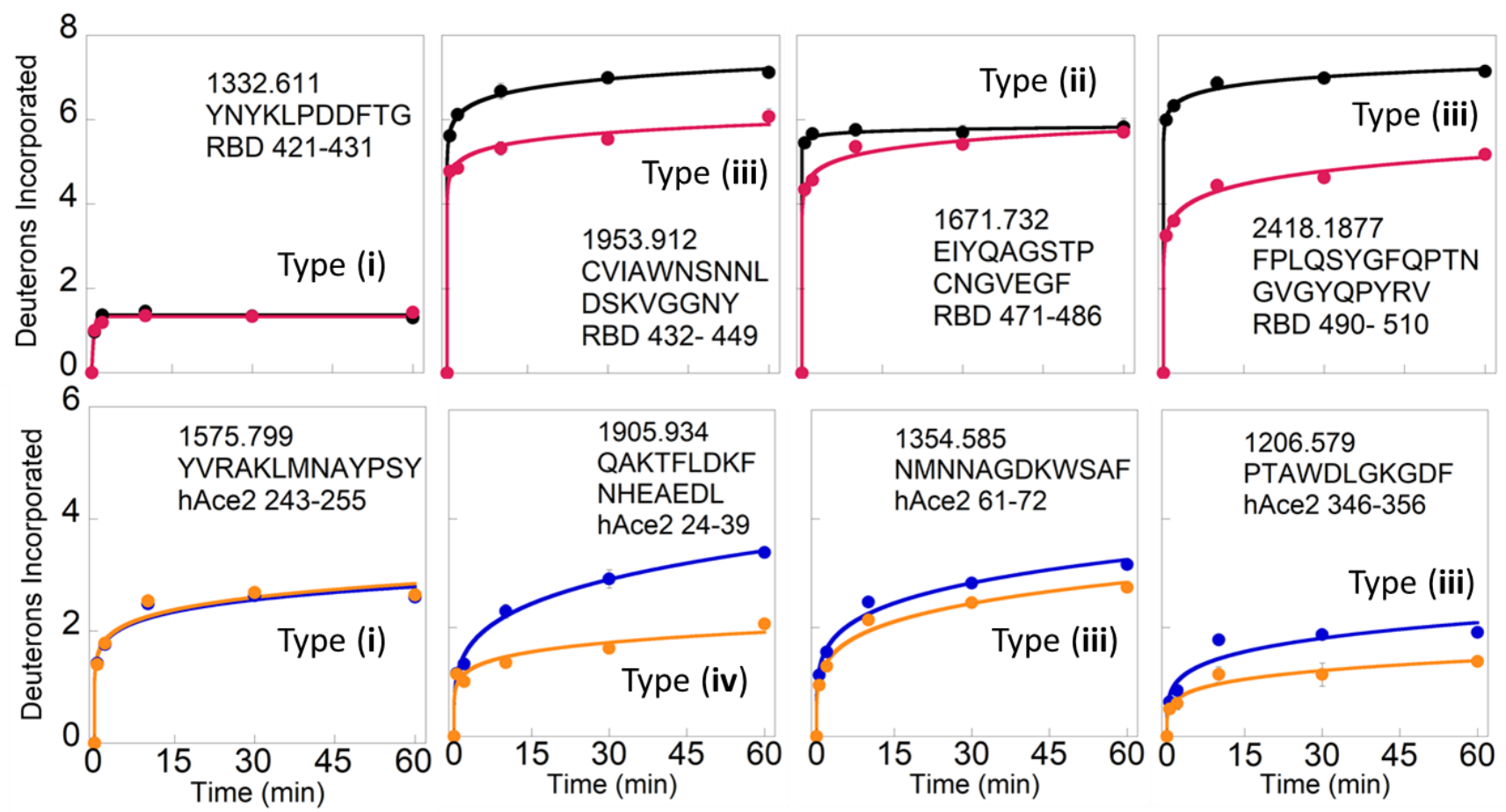

Figure 3. Deuterium uptake kinetics from selected regions of SARS-CoV-2 spike RBD (top row) and human ACE2 (bottom row). Black and blue traces correspond to free state of RBD and ACE2, respectively. Red and orange traces correspond to bound spike RBD and ACE2, respectively. Archetypal kinetic difference types are noted (see text). 
Figure 4
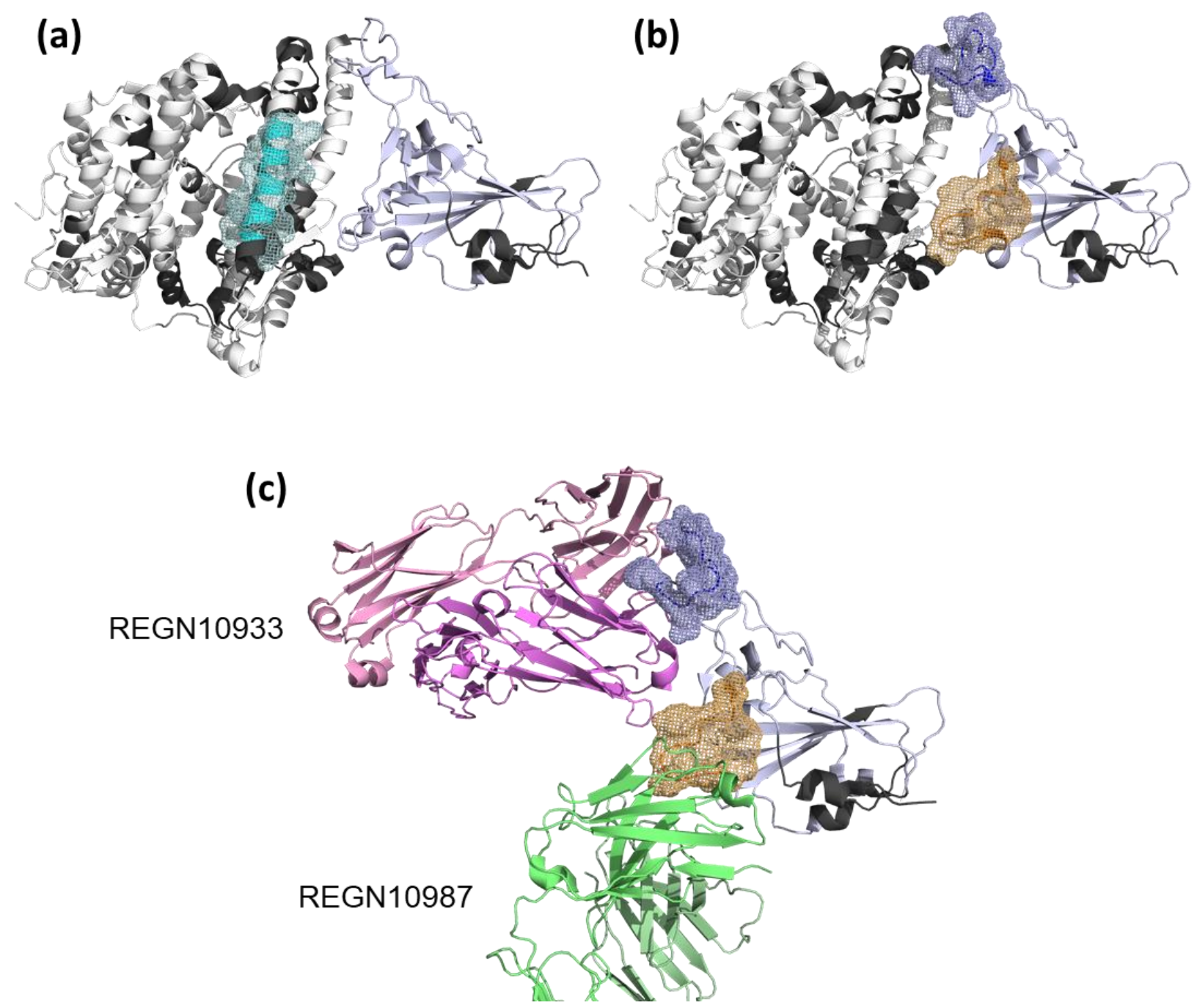

Figure 4. Conformational and dynamic changes detected outside of the core interfacial region. (a) Peptide $59-73$ of ACE2 labeled in cyan exhibits a type (iii) difference in uptake kinetics, suggesting a structural rearrangement that substantially stabilizes the $\alpha$-helix in this region. (b) Peptides $442-452$ and $471-486$ of the spike RBD exhibit type (ii) differences in uptake kinetics, suggesting a change in conformational dynamics in these regions. (c) Neutralizing antibodies REGN10933 and $10987^{27}$ bind specifically to the regions identified in (b). Structures (a) and (b) are derived from PDB $6 \mathrm{LZG}^{15}$. The structure in (c) is adapted from Hansen et al. $^{27}$ 